\title{
Magnetic Cloud as a Dual-Polarity Flux Rope
}

\author{
M. Vandas \\ Astronomical Institute, Academy of Sciences, Bočni II 1401, \\ 14131 Praha 4, Czech Republic
}

A. Geranios

Physics Department, Nuclear and Particle Physics Section, Athens University, Panepistimioupoli-Kouponia, Athens 15771, Greece

\begin{abstract}
The magnetic cloud of November 17-18, 1975 is analyzed and it is shown that measurements of magnetic field and solar wind plasma are consistent with the interpretation that this magnetic cloud is a dual-polarity flux rope which consists of a core and an annular region. The core has a strong magnetic field; the annular region has a higher plasma density and opposite magnetic polarity.
\end{abstract}

\section{Introduction}

Magnetic clouds are considered to be large interplanetary magnetic flux ropes, originated at the Sun during coronal mass ejections. Their interplanetary manifestations have been defined by Klein \& Burlaga (1982): a higher magnetic field magnitude and a lower proton temperature than in the background solar wind, and a large scale smooth rotation of the magnetic field vector of the order of one day. An example of a magnetic cloud observation is shown in Figure 1a.

\section{Analysis}

The magnetic cloud of November 17-18, 1975 (Figure 1a) has been identified by Klein \& Burlaga (1982). Burlaga (1988) suggested to approximate magnetic fields in magnetic clouds locally by constant alpha force-free fields in a cylinder (Figure 2a). The standard location of boundaries (Figure 2a) in the model cloud gives the maximum magnetic field vector rotation about $180^{\circ}$. Burlaga (1988) notes that our analyzed cloud shows a nearly $360^{\circ}$ rotation (see, within the boundaries (1)-(2), a full sinusoidal profile of the $B_{y}$ component in Figure 1a in contrary to Figure 2a) and suggests that maybe the magnetic field structure extends beyond these standard model boundaries. Figure $2 \mathrm{~b}$ illustrates this idea. Now also the model cloud, within the boundaries (1)-(2), has a full sinusoidal profile of the $B_{y}$ component. But at the boundaries (3) and (4) the axial component reverses and we have a dual-polarity flux rope (Figure 3a).

There is a remarkable drop in plasma density inside the magnetic cloud (Figure 1a), accompanied by a magnetic field increase. We shall call this region 


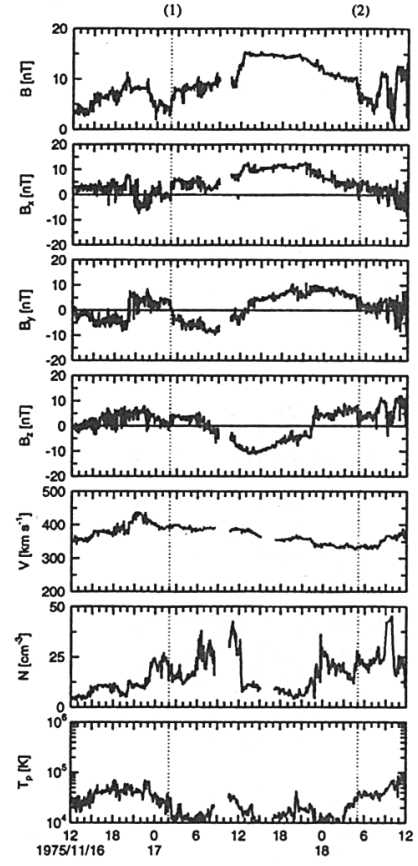

(a)

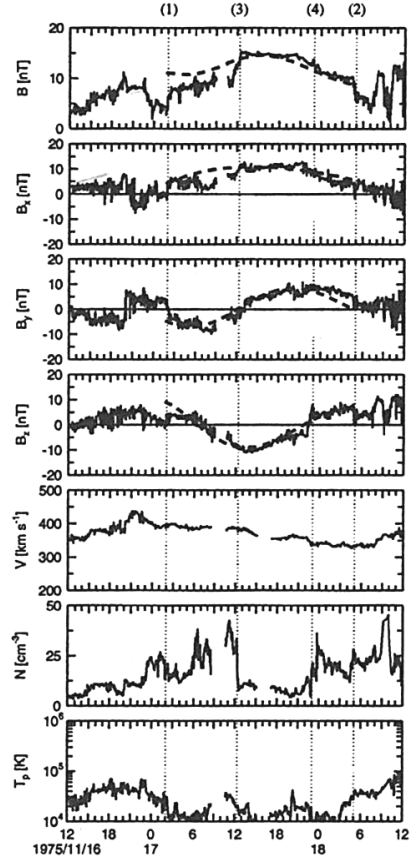

(b)

Figure 1. (a) Magnetic cloud observed by IMP 8 during November 17-18, 1975. The magnetic field magnitude $B$, and the magnetic field components in the solar ecliptic coordinates $B_{x}, B_{y}$, and $B_{z}$ are 15.36-s averages; the solar wind velocity $V$, the density $N$, and the proton temperature $T_{p}$ are 1-min averages. The dotted vertical lines labeled (1) and (2) are suggested cloud boundaries by Klein \& Burlaga (1982).

(b) The same observations but supplemented by additional boundaries (3) and (4), and by a model fit drawn in the dashed lines.

a core and identify it with one polarity region. The remaining part is an annular region with the opposite polarity and a higher density. Figure $1 \mathrm{~b}$ shows a fit of the observations by the structure from Figure $2 \mathrm{~b}$. The cloud consists of the core (3)-(4) and the denser annular region (1)-(3) \& (4)-(2). Figure 3 schematically illustrates such a configuration. Rooted at the Sun, the cloud would have magnetic field polarities at the roots like shown in Figure $3 \mathrm{~b}$.

\section{Conclusion}

We suggest that the magnetic cloud of November $17-18,1975$ is a dual-polarity flux rope consisting of the core and the annular region, which have the same magnetic chirality but opposite polarities. The core has a stronger magnetic field, while the annular region has a higher plasma density. 
(1)

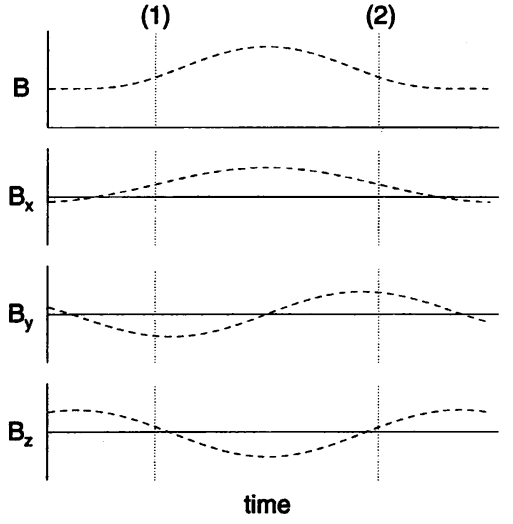

(a)

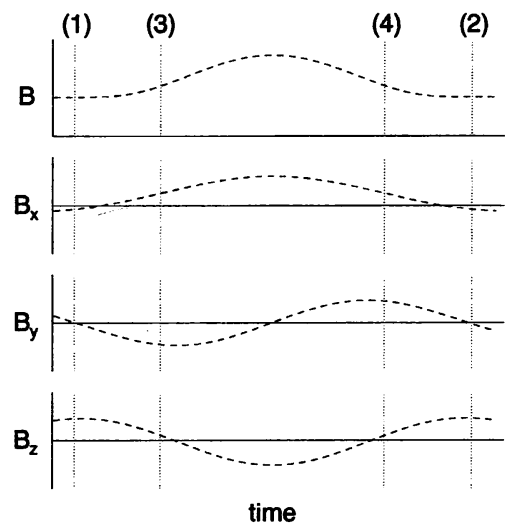

(b)

Figure 2. Model magnetic field observations of a cylindrical flux rope with constant alpha force-free fields (Burlaga 1988). (a) Boundaries of the model cloud are usually set at the places (1) and (2) where the axial component vanishes. (b) The boundaries (1) and (2) of the model cloud are extended beyond the former boundaries (labeled (3) and (4) here) to match a larger rotation of the magnetic field vector. The boundary numbers correspond to the numbers in Figure 1.

Acknowledgments. This work was supported by grant 205/99/1712 from GA ČR, and by projects ME183 from MŠMT ČR and S1003006 from AV ČR. The observational data were provided by the computer services of the National Space Science Data Center (WDC-A-R\&S).

\section{References}

Burlaga, L. F. 1988, JGR, 93, 7217

Klein, L. W., \& Burlaga, L. F. 1982, JGR, 87, 613

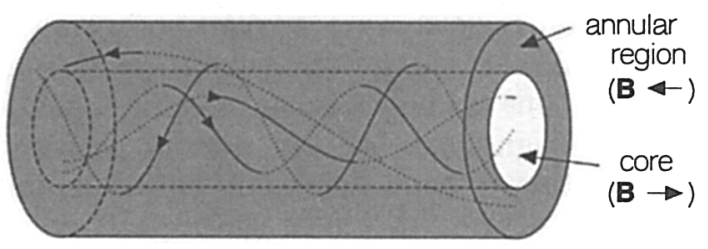

(a)

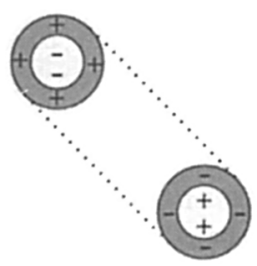

(b)

Figure 3. (a) Dual-polarity flux rope consists of the core and the annular region, which have the opposite magnetic polarities. The annular region is denser in our case; the higher density is stressed by shading. (b) Magnetic polarities at roots of a loop-like dual-polarity flux rope. 